\title{
Regulation of carotenoid and bacteriochlorophyll biosynthesis genes and identification of an evolutionarily conserved gene required for bacteriochlorophyll accumulation
}

\author{
Gregory A. Armstrong, ${ }^{*}$ David N. Cook, Dzwokai Ma, Marie Alberti, \\ Donald H. Burke and John E. Hearst \\ Department of Chemistry and Laboratory of Chemical Biodynamics, University of California, Berkeley, \\ CA 94720, USA
}

(Received 30 October 1992; revised 13 December 1992; accepted 1 February 1993)

\begin{abstract}
The temporal expression of ten clustered genes required for carotenoid (crt) and bacteriochlorophyll (bch) biosynthesis was examined during the transition from aerobic respiration to anaerobiosis requisite for the development of the photosynthetic membrane in the bacterium Rhodobacter capsulatus. Accumulation of crtA, $\operatorname{crt} C, \operatorname{crt} D, \operatorname{crt} E, \operatorname{crt} F, \operatorname{crt} K, b c h C$ and $b c h D$ mRNAs increased transiently and coordinately, up to 12-fold following removal of oxygen from the growth medium, paralleling increases in mRNAs encoding pigment-binding polypeptides of the photosynthetic apparatus. The $\operatorname{crt} B$ and $\operatorname{crt} I$ genes, in contrast, were expressed similarly in the presence or absence of oxygen. The regulation patterns of promoters for the $\operatorname{crt} A$ and $\operatorname{crt} I$ genes and the $b c h C X Y Z$ operon were characterized using $l a c Z$ transcriptional fusion and qualitatively reflected the corresponding mRNA accumulation patterns. We also report that the bchI gene product, encoded by a DNA sequence previously considered to be a portion of $\operatorname{crt} A$, shares $49 \%$ sequence identity with the nuclear-encoded Arabidopsis thaliana Cs chloroplast protein required for normal pigmentation in plants.
\end{abstract}

\section{Introduction}

Carotenoids play a variety of biological roles in both carotenogenic and noncarotenogenic organisms. These pigments protect against the destructive effects of photosensitizing molecules, notably porphyrin derivatives, and against singlet oxygen (Krinsky, 1971). In photosynthetic organisms, all of which synthesize carotenoids, these pigments also supplement the lightharvesting capacity of chlorophyll (Chl) and bacteriochlorophyll (Bchl) (Cogdell \& Frank, 1987). Recent findings implicate the carotenoid zeaxanthin in photosynthetic energy dissipation (Demmig-Adams, 1990). The general biosynthetic pathways of carotenoids and $\mathrm{Chl} / \mathrm{Bchl}$ are described in detail elsewhere (Goodwin, 1980; Castelfranco \& Beale, 1983).

The carotenoid and Bchl pigments are bound noncovalently to the polypeptides of the photosynthetic reaction centre (RC), and light-harvesting I and II (LH

*Author for correspondence. Present address: Institute for Plant Sciences, Department of Plant Genetics, ETH-Zentrum, CH-8092 Zürich, Switzerland. Tel. 4112563700 ; fax 4112520829 ; email armstrong@aeolus.ethz.ch
I and LH II, or B875 and B800-850, respectively) antenna complexes of purple non-sulphur photosynthetic bacteria, such as Rhodobacter species (Kiley \& Kaplan, 1988). The RC polypeptides are encoded by the pufL, puf $M$ and puhA genes, the LH I polypeptides by the pufA and pufB genes, and the peripheral antenna LH II polypeptides by the pucA and $p u c B$ genes in $R$. capsulatus (Scolnik \& Marrs, 1987). The puf and puh operons and all identified genes required for carotenoid $(c r t)$ and Bchl (bch) biosynthesis comprise a $46 \mathrm{~kb}$ photosynthesis gene cluster in this organism (Marrs, 1981; Zsebo \& Hearst, 1984; Burke et al., 1991 $a$ ) and in the closely related $R$. sphaeroides (Coomber et al., 1990). The assignment of $\mathrm{crt}$ genes to specific biosynthetic reactions is summarized in Fig. 1, and a biosynthetic pathway linking $b c h$ genes with particular reactions in Bchl biosynthesis has been described (Biel \& Marrs, 1983) and recently amended (Yang \& Bauer, 1990; Burke et al., 1991 b).

Diverse factors, including oxygen, light and developmental state of the organism, govern the synthesis and accumulation of carotenoid pigments in plants, algae, fungi and bacteria (Bramley \& Mackenzie, 1988), although the regulatory mechanisms remain largely obscure. The regulation of pigment synthesis in purple 


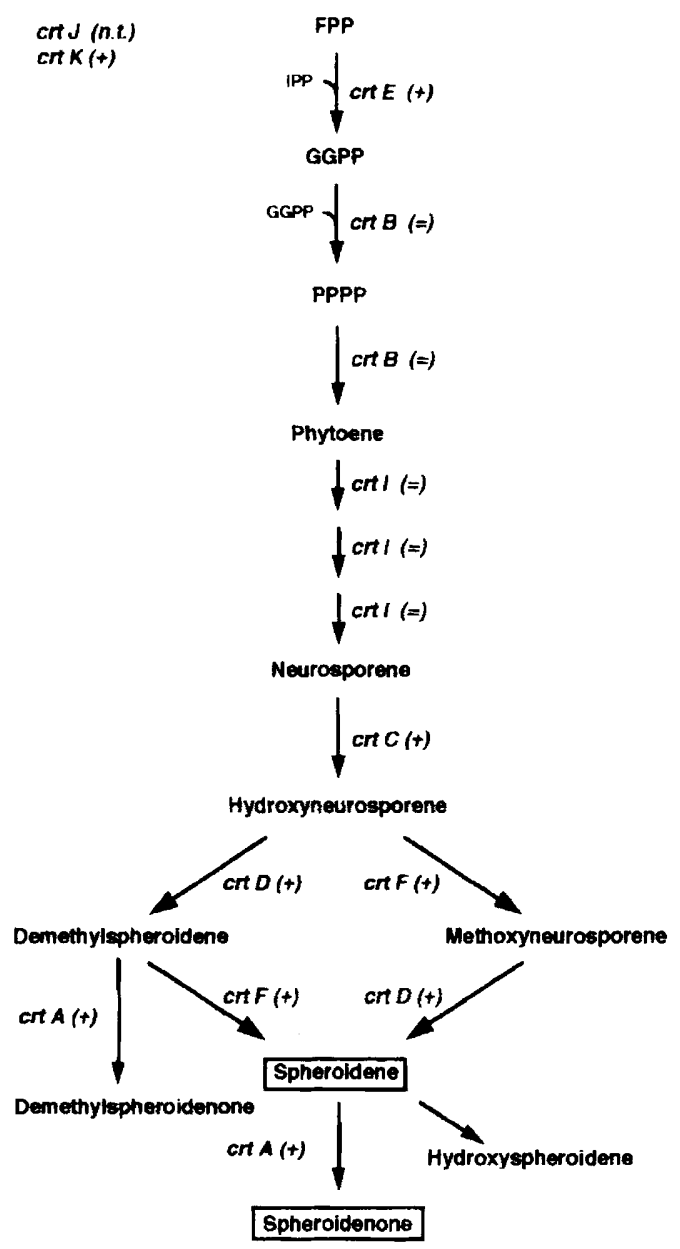

Fig. 1. The $R$. capsulatus carotenoid biosynthesis pathway. Genes encoding mRNAs that accumulate to higher levels $(+)$ or remain at constant levels $(=)$ during the onset of photosynthetic growth are indicated (see also Fig. 3). The regulation of $\mathrm{crt} J$ was not tested (n.t.). The chemical structures and semisystematic nomenclature (Straub, 1987), and a more complete description of the pathway are presented elsewhere (Armstrong et al., 1990c). IPP, FPP, GGPP and PPPP are isopentenyl pyrophosphate, farnesyl pyrophosphate, geranylgeranyl pyrophosphate, and prephytoene pyrophosphate, respectively. Phytoene is converted to neurosporene by three consecutive dehydrogenations, probably all mediated by CrtI (Bartley et al., 1990). Spheroidene and spheroidenone (boxed) are the major wild-type carotenoids accumulated in the absence and presence of oxygen, respectively. The roles of the $c r t J$ and $c r t K$ gene products in carotenoid biosynthesis are unclear. The mutation defining crtJ (Zsebo \& Hearst, 1984) and data from an in vitro carotenoid biosynthesis system (Armstrong et al., 1990 c) suggest a blockage early in the pathway or, alternatively, a regulatory mutation. The involvement of $\mathrm{CrtK}$ in carotenoid biosynthesis was postulated on the basis of the neurosporene-accumulation phenotype resulting from a mutation (Giuliano et al., 1988), later identified from the nucleotide sequence as defining a new gene (crtK) (Armstrong et al., 1989). Interestingly, it has been recently been reported that $\mathrm{CrtK}$ is homologous to a mammalian mitochondrial peripheral-type benzodiazepine receptor capable of binding protoporphyrins with nanomolar affinities (Baker \& Fanestil, 1991). Although we had tentatively proposed that $\mathrm{CrtE}$ was involved in the conversion of PPPP to phytoene (Armstrong et al., 1990 a) based on data from an $R$. capsulatus in vitro carotenoid biosynthesis system (Armstrong et al., 1990c), recent evidence demonstrates that photosynthetic bacteria has been a topic of research for almost 50 years (van Niel, 1944). Both oxygen and light intensity influence the synthesis of carotenoids, Bchl and the intracytoplasmic membrane harbouring the photosynthetic pigment-protein complexes in facultative photosynthetic bacteria such as Rhodobacter species (Cohen-Bazire et al., 1957; Kiley \& Kaplan, 1988). Either the elimination of oxygen from the growth medium accompanied by illumination of a previously dark-grown culture, or a downshift in light intensity during constant anaerobiosis, stimulates carotenoid accumulation in $R$. capsulatus (Golecki et al., 1980; Kaufmann et al., 1982) and $R$. sphaeroides (CohenBazire et al., 1957). Lowering the oxygen tension in darkgrown aerobic cultures of $R$. capsulatus produces a similar result (Schumacher \& Drews, 1978; Biel \& Marrs, 1985). Several studies report relatively little regulation of mRNA accumulation for the $R$. capsulatus crt genes during the transition from dark aerobic respiration to low oxygen conditions (Clark et al., 1984; Klug et al., 1985), or of $c r t$ promoter activities (Young et al., 1989) and mRNA levels (Zhu \& Hearst, 1986) in comparisons between steady-state dark aerobic and anaerobic photosynthetic cultures. Other experiments indicate differences in mRNA levels for some crt genes in comparisons of illuminated steady-state anaerobic and aerobic cultures (Giuliano et al., 1988). These results contrast with the strong regulation of mRNA levels and promoter activities of genes ( $p u f, p u h, p u c$ ) encoding structural pigment-binding polypeptides of the photosynthetic membrane (Clark et al., 1984; Klug et al., 1985; Zhu \& Hearst, 1986; Cook et al., 1989; Young et al., 1989).

The recent molecular characterization of carotenoid biosynthesis genes from R. capsulatus (Bartley \& Scolnik, 1989; Armstrong et al., 1989, 1990 b, c) provides the basis for more detailed studies of the regulation of individual crt genes by environmental factors. We expect that this approach will lead to a more complete understanding of the mechanisms regulating the entire biochemical pathway.

We have determined the kinetics of mRNA accumulation for eight $R$. capsulatus crt and two flanking $b c h$ genes during the well-defined transition from aerobic respiration to anaerobic photosynthesis using, for the first time, gene-specific probes. These genes form a subcluster within the photosynthesis gene cluster. We have also examined oxygen regulation of promoters for

prokaryotic homologues of CrtE and CrtB function in GGPP synthesis and catalyse the conversion of GGPP to phytoene, respectively (Chamovitz et al., 1992; Sandmann \& Misawa, 1992; Math et al., 1992). 
the divergently transcribed $c r t A$ and $c r t I$ genes, and for the better characterized $b c h C X Y Z$ operon (Wellington \& Beatty, 1989, 1991; Burke et al., 1991 b) using plasmidborne lac $Z$ ( $\beta$-galactosidase) transcriptional fusions.

\section{Methods}

Plasmid constructions. pZM400, a promoter probe vector suitable for stably maintaining lac $Z$ transcriptional fusions in $R$. capsulatus (Ma et al., 1993), was constructed by modification of pRK290, a broad host range low copy number plasmid containing an RK2 replicon (Ditta et al., 1980). Briefly, the tetracycline resistance gene of pRK290 was inactivated by introduction of a kanamycin resistance gene from $\operatorname{Tn} 903$ (Oka et al., 1981; Vieira \& Messing, 1982), and a multiple cloning site was inserted $5^{\prime}$ to a promoter-less $l a c Z$ gene from Escherichia coli. The translational start of $l a c Z$ was modified by inserting stop codons in all three reading frames upstream of a ribosome binding site, based on that found $5^{\prime}$ to the translation initiation codon of the $R$. capsulatus puhA gene. A modified version of the puc transcription terminator containing four consecutive T residues at its $3^{\prime}$ end (Chen et al., 1988) was placed $5^{\prime}$ to the multiple cloning site to minimize background expression of lac $Z$ by preventing readthrough from vector sequences. A more detailed description of pZM400 will be presented elsewhere (Ma et al., 1993). The common promoter region of the divergently transcribed $R$. capsulatus crtA and $c r t I$ genes was cloned as either a BstEII/BalI or as a $B s t E I I / S p h I$ restriction fragment (Fig. 3) into the pZM400 multiple cloning site. The resulting plasmids, pZM422 and pZM432, contain $R$. capsulatus DNA in the appropriate orientations to place $\operatorname{cr} I$ and $\operatorname{crt} A$ promoter sequences, respectively, $5^{\prime}$ to lac $Z$. The $R$. capsulatus bch $C X Y Z$ promoter region was inserted into the pMZ400 multiple cloning site as a SmaI/ApaI fragment (Fig. 3), yielding pZM410. Molecular biological manipulations were carried out using standard protocols supplied by manufacturers.

Growth and manipulation of bacterial strains, isolation of $R N A$ and measurement of LacZ activities. R. capsulatus strain SB1003, wild-type for photosynthetic functions (Yen \& Marrs, 1976), was grown under the controlled conditions described by Cook et al. (1989) and below. Dark-grown aerobic cultures were sparged with a mixture of $\mathrm{N}_{2}: \mathrm{O}_{2}: \mathrm{CO}_{2}(80: 20: 2$, by vol.). Photosynthetic cultures were illuminated at $15 \mathrm{~W} \mathrm{~m}^{-2}$ by a bank of Lumiline lamps (General Electric) and sparged with a mixture of $\mathrm{N}_{2}: \mathrm{CO}_{2}(80: 2$, by vol.). At time zero of the transition from aerobic respiration to photosynthesis, the gas mixture was changed to eliminate $\mathrm{O}_{2}$ from the growth medium and the cultures were exposed to light. To determine the effect of light on gene expression, duplicate dark-grown cultures were switched from the aerobic to the anaerobic gas mixture described above, with one culture simultaneously exposed to light.

Samples $(6 \mathrm{ml})$ of the SB1003 cell cultures were removed at various times during aerobic respiratory growth or the transition to photosynthesis for RNA isolation. Samples were immediately mixed with an equal volume of ice cold buffer A ( $80 \mathrm{~mm}-\mathrm{Tris} / \mathrm{HCl}, \mathrm{pH} 7.5,10 \mathrm{~mm}-$ $\mathrm{MgCl}_{2}$ ) supplemented with $200 \mu \mathrm{g}$ chloramphenicol ml $\mathrm{m}^{-1}, 25 \mathrm{~mm}-$ sodium azide and $10 \mathrm{~mm}-\beta$-mercaptoethanol (Zhu \& Kaplan, 1985), cooled for $15 \mathrm{~s}$ in a dry ice/ethanol bath and harvested by centrifugation. The pellets were resuspended in lysis buffer, immediately frozen on dry ice and stored at $-70^{\circ} \mathrm{C}$ until isolation of the RNA as described (Zhu \& Kaplan, 1985).

pZM plasmids were introduced into $R$. capsulatus SB1003 by triparental matings with $E$. coli plasmid donor and helper strains as described (Zsebo \& Hearst, 1984). For LacZ activity measurements, exponentially-growing $R$. capsulatus cells were shifted from aerobic to photosynthetic growth as described above. Samples $(5 \mathrm{ml})$ were collected to monitor LacZ activities by the method of Miller (1972), with $0.2 \mathrm{ml}$ of cells assayed in $0.8 \mathrm{ml} \mathrm{Z}$-buffer. All $R$. capsulatus cultures were grown at $32{ }^{\circ} \mathrm{C}$.

Preparation of RNA dot-blots, M13 probes and nucleic acid hybridizations. RNA dot-blots were prepared as previously described, using $5 \mu$ g total RNA per dot (Cook et al., 1989). RNA time courses were probed for specific mRNAs with single-stranded M13 subclones, labelled by primer extension across the insert region with the Klenow fragment of DNA polymerase I as previously described (Armstrong et al., $1990 c)$, except that $5^{\prime}-\left[\alpha-{ }^{32} \mathrm{P}\right] \mathrm{dATP}$ was replaced by $50 \mu \mathrm{Ci} 5^{\prime}-$ $\left[\alpha-{ }^{32} \mathrm{P}\right] \mathrm{dCTP}\left(3000 \mathrm{Ci} \mathrm{mmol}^{-1}\right.$; Amersham). Gene-specific M13 probes for the $c r t$ genes, $b c h C$ and $b c h D$ (Fig. 3) were generated during the sequencing of these regions (Armstrong et al., 1989). The use of the gene-specific M13 probes for the puf operon (pufL) and the puh operon (puhA) (Zhu \& Hearst, 1986) to obtain the data plotted in Fig. 2(b) has been described (Cook et al., 1989), as has the gene-specific 15-mer oligonucleotide probe for the $p u c$ operon $(p u c B)$ (Zhu \& Hearst, 1986). The pucB oligonucleotide $(500 \mathrm{ng})$ was end-labelled with $50 \mu \mathrm{Ci} 5^{\prime}$ $\left[\gamma^{32} \mathrm{P}\right] \mathrm{rATP}\left(5000 \mathrm{Ci} \mathrm{mmol}^{-1}\right)$ and T4 polynucleotide kinase. To insure that equivalent amounts of RNA from each sample were applied to the membrane, we prepared dot-blots using about $3 \mathrm{ng}$ total RNA per time point and hybridized these with either radiolabelled $\mathrm{pRCl}$, containing cloned $R$. capsulatus $\mathrm{rRNA}$ genes (Yu et al., 1982), or with radiolabelled total $R$. capsulatus chromosomal DNA isolated as described previously (Cook et al., 1989). pRCl (1 $\mu \mathrm{g})$ or total chromosomal DNA $(1 \mu \mathrm{g})$ was labelled with $50 \mu \mathrm{Ci} \quad 5^{\prime}-\left[\alpha-{ }^{32} \mathrm{P}\right] \mathrm{dCTP}\left(3000 \mathrm{Ci} \mathrm{mmol}^{-1}\right)$ by nicktranslation using a Bethesda Research Laboratories kit. RNA dot-blot hybridizations with Gene Screen nylon membranes (New England Nuclear), autoradiography, and quantification of the excised dots by scintillation counting (Fig. 2) were performed as described previously (Cook et al., 1989), except that the RNAs to be hybridized with the $p u c B$ oligonucleotide probe were applied to a nitrocellulose membrane (Schleicher \& Schuell). An autoradiogram of the puc hybridization was quantitated as above (Fig. $2 b$ ). Autoradiograms of membranes probed for $\operatorname{crt} C, c r t D$ and $b c h D$ mRNAs were quantified by densitometry using a Hoefer Scientific Instruments scanning densitometer, Model GS 300 (data not shown).

Database searches and sequence alignments. Sequence similarity between the $R$. capsulatus BchI and $A$. thaliana Cs proteins was detected by searching the NBRF database (release 28.0, 3/91) using the FASTA program from the GCG software package, version 6.2 (Devereux et al., 1984). The BESTFET program from this package was used for the initial sequence alignment, which was subsequently optimized by hand.

\section{Results and Discussion}

\section{Accumulation of many crt and bch $m R N A$ s increases during the transition to photosynthesis}

During the transient cessation of cellular division that follows a rigorous transition from dark aerobic respiration to anaerobiosis in the light, Rhodobacter must adapt to photosynthetic growth through the synthesis of carotenoids, Bchl, pigment-binding polypeptides and an intracytoplasmic membrane system harbouring these components (Kiley \& Kaplan, 1988). The rates of carotenoid and Bchl synthesis increase rapidly during this lag phase (Cohen-Bazire et al., 1957; Schumacher \& Drews, 1978).

We have previously demonstrated that $R$. capsulatus cell division ceases and rRNA levels remain constant during the first hour of such a transition (Cook et al., 

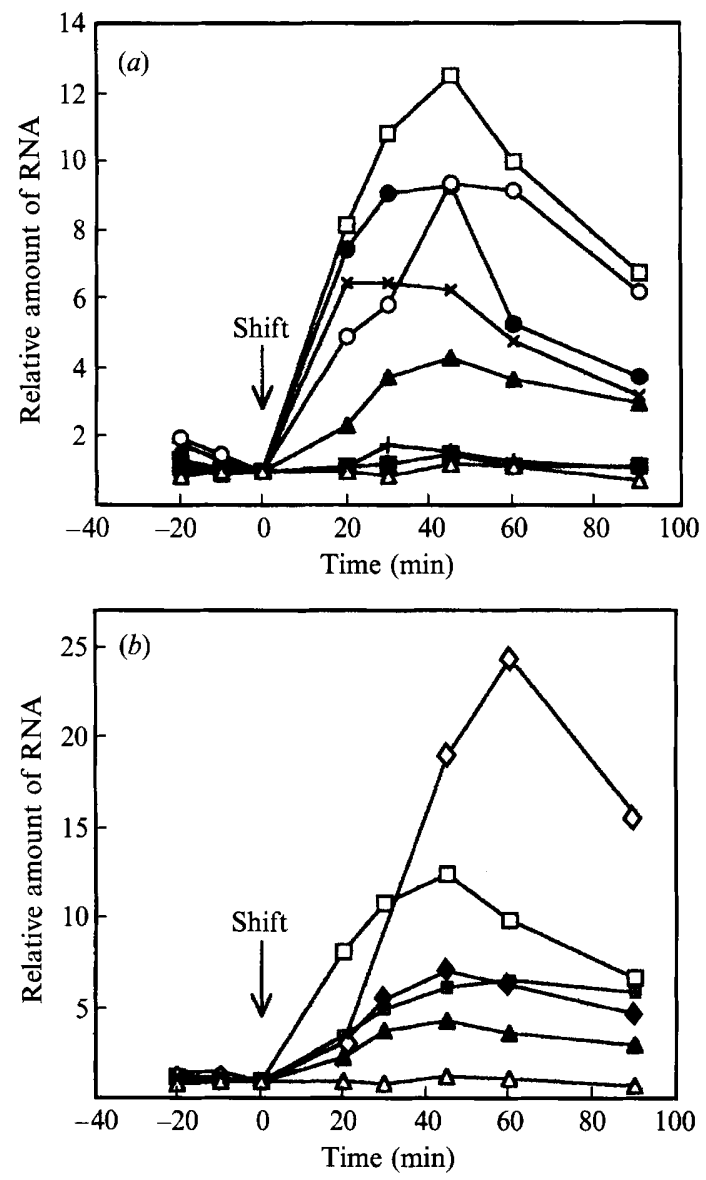

Fig. 2. Time courses and magnitudes of mRNA accumulation for $c r t$ and $b c h$ genes compared with the puf, puh, and puc mRNAs in the same experiment. Times in min are given relative to time zero when bacterial cultures were shifted from dark aerobic respiratory growth to anaerobic photosynthetic conditions. The quantification of bound radioactivity from RNA dot-blots hybridized with DNA probes is shown. (a) mRNA levels for $c r t$ and $b c h$ genes plotted relative to an arbitrary value of one at time zero for each mRNA. The probe for crtI mRNA was BSa6 (see Fig. 3 legend for exact locations of all probes used in this work). A similar hybridization pattern was also obtained using probe HH121 for crtI (data not shown). Standard deviations in the amounts of each mRNA, as determined based on the values at $-20,-10$ and 0 min, are as follows: $\operatorname{crtA}(\square), 0.068 ; \operatorname{crtB}(+), 0.350 ; \operatorname{crt} E(\mathbf{O}), 0.093$; $\operatorname{crtF}(\times), 0.320 ; \operatorname{crtI}(\mathbf{\square}), 0.171 ; \operatorname{crtK}(\mathrm{O}), 0.379 ; b c h C(\mathbf{\Delta}), 0.151$; rRNA $(\triangle), 0.061$. (b) Comparison of crtA and bchC mRNA accumulation with the accumulation of $p u f, p u h$, and $p u c$ mRNAs. RNA dot-blots probed for puf and puh mRNAs (Cook et al., 1989) and puc mRNA, with specific DNA probes for each of these sequences (Zhu \& Hearst, 1986), were used for this quantitative presentation. Symbols and standard deviations in the amounts of specific mRNAs not given in $(a)$, as determined based on the values at $-20,-10$ and 0 mins, are as follows: pufL $(\boxplus), 0.086 ; p u h A(\diamond), 0.076 ; p u c B(\diamond), 0.198$.

1989). In this experiment we also determined mRNA accumulation patterns for the crt and two bch genes during the adaptive period (Fig. 2). Probes derived from an rDNA sequence or total genomic DNA were used to demonstrate that equivalent amounts of RNA for each time point had been applied to the membrane (Fig. 2 and data not shown). mRNA levels for all $c r t$ and $b c h$ genes remained constant prior to the simultaneous removal of oxygen from the growth medium and illumination of the cultures.

The crt and bch mRNAs fall into two groups depending on their response to this change in environmental conditions. The accumulation of mRNAs for $\operatorname{crt} A, \operatorname{crt} C$ (not shown), $c r t D$ (not shown), $\operatorname{crt} E, \operatorname{crt} F$, $c r t K, b c h C$ and $b c h D$ (not shown) increased 2- to 12-fold, while mRNA levels for $\operatorname{crt} B$ and $c r t I$ remained constant (Fig. $2 a$ ). In the same experiment, accumulation of $p u f$ and puh (LH I and RC) mRNAs increased comparably (6- to 8-fold; Cook et al., 1989), while puc (LH II) mRNA increased 25-fold (Fig. 2b). The results of the $\mathrm{crt}$ and $b c h$ mRNA accumulation studies and the locations of the DNA probes used are summarized in Fig. 3.

Our results thus indicate that regulated $c r t$ and $b c h$ mRNA accumulation may play as significant a role in the expression of pigment biosynthesis enzymes as in the expression of pigment-binding polypeptides during the rigorous transition from dark aerobic respiration to anaerobic photosynthesis. Three important features combined in this study distinguish it from previous efforts, discussed below, in this area: (i) the use of genespecific probes for each mRNA studied, including mRNAs representing both pigment biosynthetic enzymes and pigment-binding polypeptides, (ii) the measurement of both mRNA accumulation and promoter activities using identical and well-defined growth conditions to obtain two independent measures of gene regulation, (iii) the detailed sampling of time points during the early stages of the adaptation to photosynthetic growth.

Several earlier studies, including work from this laboratory, had indicated relatively small changes in $R$. capsulatus crt mRNA levels (Clark et al., 1984; Klug et al., 1985; Zhu \& Hearst, 1986) or promoter activities (Young et al., 1989) in response to low oxygen tensions or anaerobic photosynthetic conditions, in contrast to the puf operon. Discrepancies between our results and earlier comparative studies of mRNA accumulation probably result in large part from the previous unavailability of gene-specific probes for crt mRNAs and the absence, with one exception (Clark et al., 1984), of detailed kinetic data. Differences in the experimental techniques, bacterial strains, or the growth conditions used could also be contributing factors. puf and puc mRNAs, for example, accumulate to significantly higher levels in dark-grown $R$. capsulatus cultures shifted from $20 \%$ to $3 \%$ oxygen tension (Leach et al., 1991) than in cultures shifted to completely anaerobic conditions (Fig. $2 b$; Cook et al., 1989).

Some evidence for the regulation of certain $\mathrm{crt}$ mRNAs was obtained by comparing anaerobic versus aerobic 

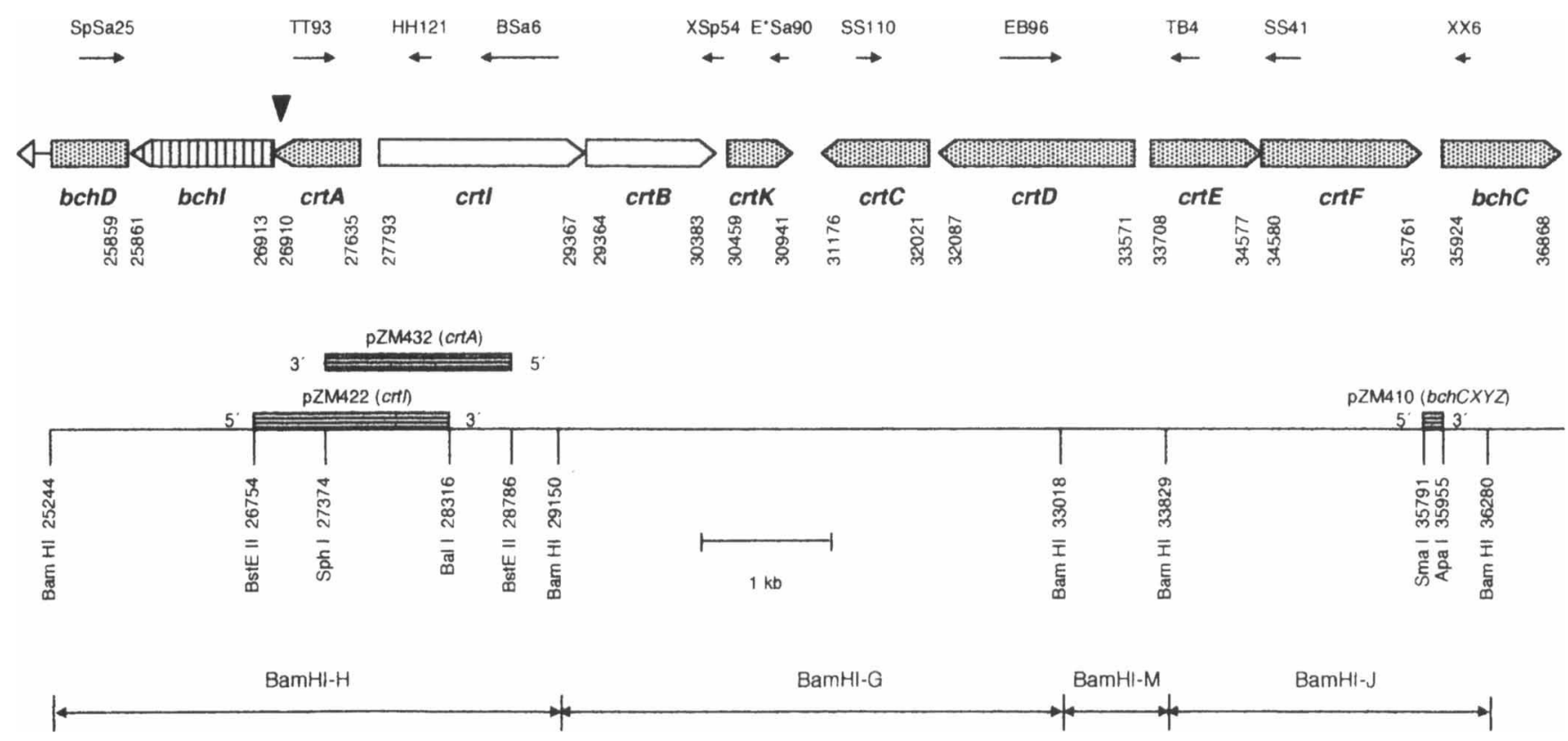

Fig. 3. Genetic-physical map of the $R$. capsulatus carotenoid biosynthesis gene cluster. The scale at the bottom shows the locations of all BamHI restriction sites. The Bam HI-H, -G, -M and -J restriction fragments of the $46 \mathrm{~kb}$ photosynthesis gene cluster carried on pRPS404 (Marrs, 1981; Burke et al., 1991 a) have been used as probes in several previous studies of $\mathrm{crt}$ gene expression (Clark et al., 1984; Klug et al., 1985; Zhu \& Hearst, 1986). Orientations of the genes are as indicated. bchI (see Fig. 5 and text) was previously considered to be a portion of $c r t A$ (Armstrong et al., 1989, 1990 b c c). The detached arrowhead indicates that $b c h D$ extends into the flanking BamHI-E restriction fragment. Other selected restriction sites used in the construction of $R$. capsulatus promoter-lac $Z$ transcriptional fusion plasmids (pZM410, bchCXYZ; pZM422, crtI; pZM432, crtA) are indicated. The extents and orientations of the $R$. capsulatus DNA inserted $5^{\prime}$ to the $l a c Z$ gene in these plasmids are indicated by rectangles with horizontal stripes. The nucleotide positions of the genes are given from the first nucleotide of the start codon to the last nucleotide of the stop codon (vertical numbers) (Burke et al., 1991 a). The numbering system used in the original publication describing this sequence (Armstrong et al., 1989) can be obtained by subtracting 25243 from the nucleotide positions given here. Gene-specific single-stranded M13 probes used to detect mRNAs from different regions of the gene cluster are indicated above, with arrows showing the direction of the synthesized probe and the extent of the insert. The exact map locations of the M13 probes used are as follows: SpSa25 (bchD), 25470-25812; TT93 (crtA), 27 105-27436; HH121 (crtI), 27997-28 157; BSa6 (crtI), 28 544-29150; XSp54 (crtB), 30250-30418; E*Sa90 (crtK), 30826-30892; SS110 (crtC), 31446-31651; EB96 (crtD), 32553-33018; TB4 (crtE), 33830-34060; SS41 (crtF), 34576-34842; XX6 (bchC), 35994 36085. mRNA accumulation during photosynthetic adaptation is indicated by shading of the genes: grey, 2-to 12-fold increase; white, no increase; vertical stripes, not tested. The black triangle indicates the position of a frameshift mutation in the previously reported $\operatorname{crt} A$ sequence (see Fig. 5 legend).

cultures under constant illumination (Giuliano et al., 1988), a condition which allows Rhodobacter species to use both photosynthetic and respiratory growth modes simultaneously (Cohen-Bazire et al., 1957; Keister, 1978), Our quantitative data on the temporal regulation of mRNA accumulation (Fig. 2a) do, despite the difference in growth conditions used, agree with the qualitative steady-state results previously reported (Giuliano et al., 1988). The measured increases in $\mathrm{crt} E$ and $\operatorname{cr} F \mathrm{mRNAs}$ during the transition to photosynthesis (Fig. 2a) also agree with the oxygen regulation attributed to an inducible promoter for the crtEF operon using plasmid-borne LacZ translational fusions (Young et al., 1989).

The constitutive patterns of $c r t I$ and $\operatorname{crt} B$ mRNA expression detected using three different DNA probes (Fig. 3) are consistent with the proposed cotranscription of these genes (Giuliano et al., 1988; Armstrong et al.,
1989). In contrast, $c r t K$ mRNA accumulation increased 8 -fold during the shift from aerobic respiration to photosynthetic growth (Fig. 2a), suggesting that $\mathrm{crtK}$ may form a distinct operon. An alternative explanation would be the differential regulation of mRNA stability within the same transcript, as documented for the $R$. capsulatus puf operon (Belasco et al., 1985; Chen et al., 1988).

Although changes in light intensity during photosynthetic growth can influence carotenoid content in Rhodobacter species (Cohen-Bazire et al., 1957; Golecki et al., 1980; Kaufmann et al., 1982), pigment accumulation can also be triggered solely by a downshift in oxygen tension in dark-grown cultures (Schumacher \& Drews, 1978; Biel \& Marrs, 1985). To address directly the possible regulation of $R$. capsulatus crt genes by light we examined the expression of $\operatorname{crtA}$, a gene strongly regulated by oxygen tension, during the transition from 
dark aerobic respiration to either dark anaerobic or anaerobic photosynthetic conditions. Cells shifted in the presence of light displayed a transient 10 -fold increase in crtA mRNA, versus a 5-fold increase in the absence of light (data not shown). The patterns of mRNA accumulation versus time were equivalent in both of these experiments to the results presented in Fig. 2. Thus, the removal of oxygen from the growth medium suffices qualitatively for the induced accumulation of $\operatorname{crt} A$ mRNA, although the presence of light may play a modulating role.

mRNAs for different classes of genes required for photosynthesis accumulate coordinately

mRNAs for all regulated crt genes, and for $b c h C$ and $b c h D$, accumulated coordinately during the first $45 \mathrm{~min}$ of the adaptation to photosynthetic growth (Fig. 2a). puf, puh, and puc mRNAs accumulated with time courses roughly similar to those of the regulated $\mathrm{crt}$, and $b c h$ mRNAs, although their accumulation continued up to $60 \mathrm{~min}$ into the transition, in some cases (Fig. 2b). This temporal pattern of mRNA accumulation may reflect a coordinated programme of gene expression during the development of a functional photosynthetic membrane. The stable assembly of the pigment-protein complexes relies on the presence of the pigments in $R$. capsulatus (Zsebo \& Hearst, 1984; Klug et al., 1985), suggesting the biological significance of such an expression pattern.

One mechanism for a rapid and transient increase in carotenoid and Bchl production to meet the demands of the cell would be the accumulation of those mRNAs encoding enzymes governing rate-limiting reactions in the respective biosynthetic pathways (Fig. 1; see Biel \& Marrs (1983) for an outline of the Bchl biosynthetic pathway). The intracellular levels of CrtI are apparently not a limiting factor in carotenoid biosynthesis (Bartley \& Scolnik, 1989). Consistent with this, we did not observe regulation of crtI mRNA accumulation or transcriptional activity. Although the different $R$. capsulatus carotenoid biosynthesis enzymes have not yet been biochemically characterized and their relative enzymic activities have not been measured, the constitutive expression of $\operatorname{crt} B$ and $\operatorname{crtI} \mathrm{mRNAs}$ indicates that biosynthetic capacity for the conversion of GGPP to neurosporene (Fig. 1) is not regulated at the level of mRNA. The regulated crt genes encode products required both early in carotenoid biosynthesis (before GGPP) and late in the pathway (after neurosporene). The increase in crtE expression could, for example, reflect an increased requirement for GGPP in the synthesis of both carotenoids and of the phytyl moiety of Bchl in response to photosynthetic conditions. Expres-

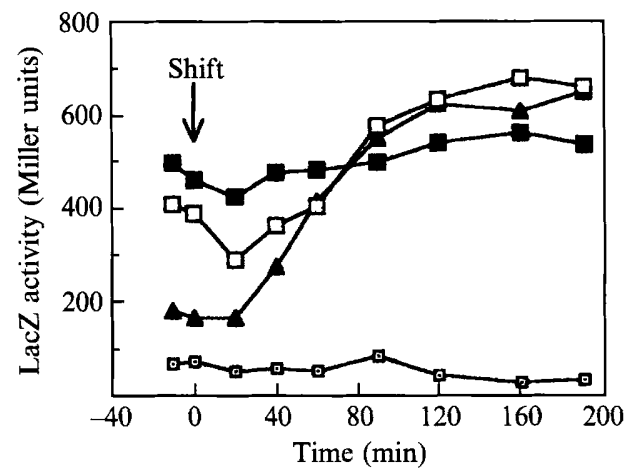

Fig. 4. Time courses of lac $Z$ expression driven by the $\operatorname{crt} A, c r t I$ and $b c h C X Y Z$ promoters during the transition to photosynthesis. Growth conditions are as described in Fig. 2. The control ( $\square$ ) is the promoterless construct, pZM400, while $b \operatorname{ch} C X Y Z(\mathbf{\Delta}), \operatorname{crtI}(\mathbf{\square})$ and $\operatorname{crt} A(\square)$ are the lac $Z$ transcriptional fusion constructs pZM410, pZM422 and pZM432, respectively. Standard deviations in the LacZ activities, as determined based on the values at -10 and $0 \mathrm{~min}$, are as follows: $\operatorname{crt} A$, 12 ; crtI, 20.5; bchCXYZ, 7; control, 3.6.

sion of a homologue of $c r t E$ from another bacterium significantly increases GGPP accumulation in E. coli (Sandmann \& Misawa, 1992).

\section{Oxygen regulation of crtI, crtA and bchCXYZ promoter activities determined using lac $Z$ transcriptional fusions}

The oxygen regulation of $\operatorname{crtI}, \operatorname{crt} A$, and $b \operatorname{ch} C X Y Z$ expression was further studied by fusion of promotercontaining restriction fragments (Fig. 3) $5^{\prime}$ to a promoterless $E$. coli lac $Z$ gene resident in the low copy number plasmid pZM400. The crtI, crt $A$ and $b c h C X Y Z$ promoter constructs, pZM422, pZM432 and pZM410, respectively, were introduced into $R$. capsulatus strain SB1003. Cells were assayed for LacZ activity at different times during the adaptation to photosynthetic growth under the same conditions used to measure mRNA accumulation.

When $R$. capsulatus cultures were shifted from dark aerobic growth to anaerobic photosynthetic conditions, LacZ activity driven by the $\operatorname{cr} t A$ and $b c h C X Y Z$ promoter regions increased 2- and 3.5-fold, respectively, reaching a plateau approximately $60 \mathrm{~min}$ after the maximal mRNA accumulation (Fig. 4). This lag may reflect the translation of the lac $Z$ mRNA and the gradual accumulation of active LacZ enzyme. crtI promoter-driven LacZ activity remained roughly constant during the same time course, in agreement with the RNA data. The background level of LacZ activity from the promoter-less control, pZM400, was an order of magnitude lower than that observed in the promoter-containing constructs under photosynthetic conditions. The difference between the increases in $\operatorname{cr} A$ promoter activity (two-fold; Fig. 4) and 
1577 TGTCTTCCTGACCGACGATGGCAGAAAAGGGAAAGACGGGTCTCGTCTTCGCGCCCGAAGCAGAGGGTTGAAGTCGAGCGACGGCGGTAGTCATGCAGTTTCCCTTTCCAAGACTTTGCT ACAGAAGGACTGGCTGCTACCGTCTTTTCCCTTTCTGOCCAGAGCAGAAGCGCGGGCTTCGTCTCCCAACTTCAGCTCGCTGCCGCCATCAGTACGTCAAAGGGAAAGGTTCTGAAACGA

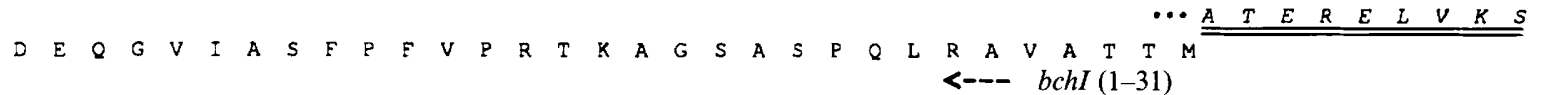

1697 GCCGAGCGGGTCCTTGCCCATCCAGCTTCCCTCGGT \#CCAAGCAAACGGAACCGGGCATAGAGTTCTTCGGTGAACCAACCTTCTTCACGCGCAGCACGGATGCCCTTGCCATGCGGCG CCGCTCGCCCAGGAACGGGTAGGTCGAAGGGAGCCA\#GGGTTCGTTTCCCTTGGCCCGTATCTCAAGAAGCCACTTGGTTGGAAGAAGTGCGCGTCGTGCCTACCGGAACGGTACGCCGC

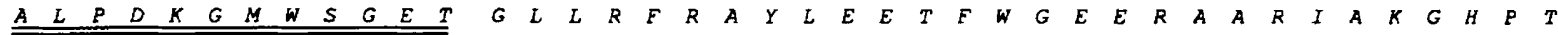
<-- $\operatorname{crtA}(193-241)$

Fig. 5. The revised nucleotide sequence of $R$. capsulatus crtA reveals a new gene, $b c h I$, required for Bchl biosynthesis or accumulation. Nucleotide positions, indicated at the left, and the orientations of the protein coding sequences are given as in the original presentation of this nucleotide sequence in order to facilitate comparison (Armstrong et al., 1989) (EMBL database accession number X52291). * indicates the position of a frameshift error caused by insertion of an extra nucleotide in the original sequence. The corrected nucleotide sequence shown here can be obtained from the EMBL database under accession number Z11165 (Burke et al., 1991 a), starting from position 26820. Single letter amino acid translations are given for portions of BchI and CrtA (inclusive residues are shown in parentheses). Three dots indicate the new $\operatorname{crt} A$ stop codon. The putative $b c h I$ ribosome binding site is underlined. Doubly underlined amino acids in the CrtA sequence indicate new residues introduced between the frameshift and the revised stop codon.

mRNA accumulation (12-fold; Fig. 2) in response to anaerobiosis could refiect either post-transcriptional regulation, or differences in expression between the chromosomal and plasmid-borne copies of the crtA promoter due to DNA structure or plasmid copy number.

In agreement with our results, chromosomal (Biel \& Marrs, 1983) and plasmid-borne (Wellington \& Beatty, 1989, 1991; Young et al., 1989) lacZ transcriptional and translational fusions in $R$. capsulatus have indicated that the steady-state activity of the $b c h C X Y Z$ promoter (Burke et al., 1991 b) increases between 3- and 8-fold when comparing growth under high and low oxygen tensions or photosynthetic conditions with respiration. Although transcripts initiated upstream of the $b c h C X Y Z$ promoter (Fig. 3) can read through into the $b c h C$ gene (Wellington \& Beatty, 1991), the magnitude of $b c h C$ mRNA accumulation (3-fold) corresponds closely with the increase in transcription initiation from the $b c h C X Y Z$ promoter in our experiments (compare Figs 2 and 4). A thorough characterization of the $b c h C X Y Z$ promoter will be presented elsewhere (Ma et al., 1993). The data presented here on the temporal expression of $b c h C X Y Z$ promoter activity and $b c h C$ and $b c h D$ mRNAs, combined with measurements made by other researchers (Biel \& Marrs, 1983; Hunter \& Coomber, 1988; Young et al., 1989; Yang \& Bauer, 1990; Wellington \& Beatty, 1991), demonstrate moderate regulation of many $b c h$ genes by oxygen tension.

Identification of the bchI gene and conservation of the predicted gene product in more evolved photosynthetic organisms

Previous experiments with $R$. capsulatus had established that the Tn5.7 insertion mutation defining the $b c h I$ locus as an entity distinct from $b c h D$ and $\operatorname{crt} A$ by complementation (Zsebo \& Hearst, 1984) mapped physically within the $3^{\prime}$ portion of the ORF assigned to the crtA gene (Armstrong et al., 1990c). This Tn5.7 mutation abolished the accumulation of Bchl or its visibly absorbing precursors (Zsebo \& Hearst, 1984). In the course of the current study, we determined that the Cterminal half of the predicted $c r t A$ gene product displays a high degree of sequence similarity with the Arabidopsis thaliana Cs protein (Koncz et al., 1990). cs (ch-42) is a light-regulated nuclear gene which encodes a protein destined for the chloroplast and required for normal pigmentation in plants. These data led us to consider the possibility that the published nucleotide sequence of $\mathrm{crtA}$ might contain an error (Armstrong et al., 1989).

Redetermination of the nucleotide sequence of the entire $\operatorname{crt} A$ coding region revealed one change, the absence of a single nucleotide at position 1733 (Fig. 5), with respect to the original sequence. As a consequence, the long ORF of 591 amino acids thought to encode CrtA is split into two segments. 21 amino acids of the $5^{\prime}$ ORF following the frameshift error (Thr-221 to Ala-241) differ from those originally reported for CrtA before an in-frame stop codon is reached. Overlapping the stop codon, an AUG start codon preceded by a potential ribosome binding site (Fig. 5) could be used to initiate translation of a $3^{\prime}$ ORF of 350 amino acids, corresponding to residues 242 to 591 originally deduced for CrtA. In accordance with the above considerations, we propose that the $5^{\prime}$ and $3^{\prime}$ ORFs encode CrtA and BchI, respectively. Based on the gene order in this region (Zsebo \& Hearst, 1984; Burke et al., 1991a), previous data from in vivo complementation studies (Taylor et al., 1983; Zsebo \& Hearst, 1984; Giuliano et al., 1988), and the rough mapping of $b c h D$ transposon-induced mutations (Zsebo, 1984), we propose that the ORF downstream from $b c h I$ corresponds to $b c h D$. Absolute confirmation of these proposals will, however, require 


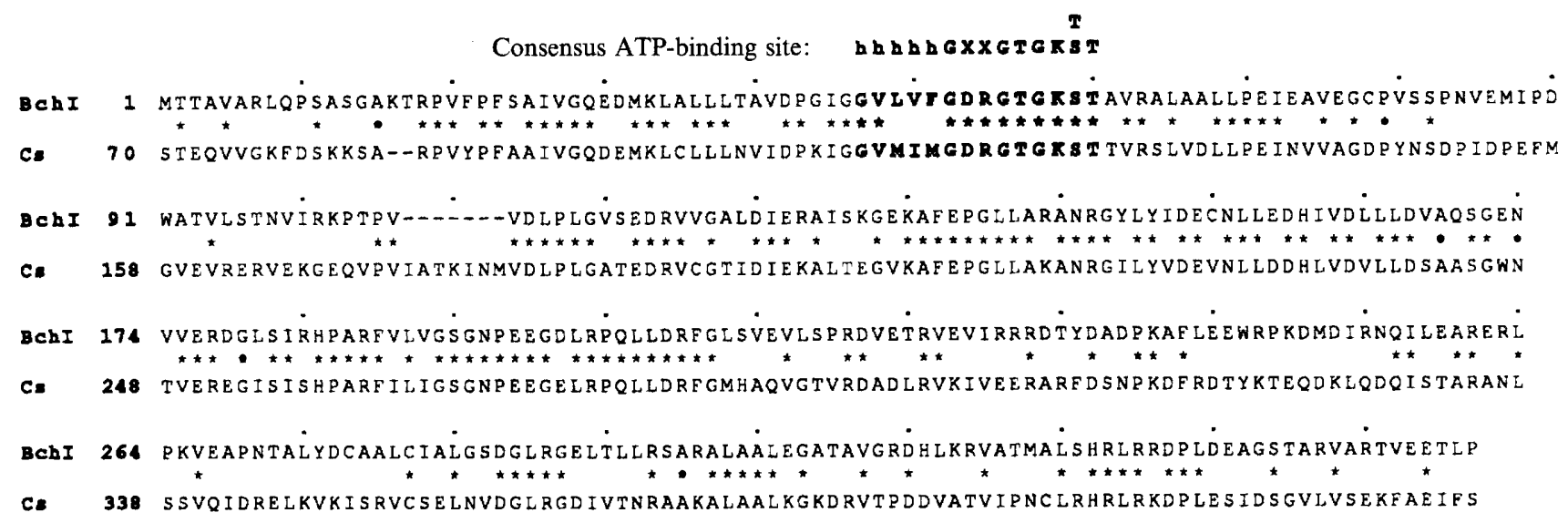

Fig. 6. The R. capsulatus BchI and A. thaliana Cs proteins are highly conserved and contain putative ATP-binding sites. Numbers at the left indicate the amino acid positions. Identities between the two sequences are shown by asterisks. Dashes indicate gaps inserted to maximize the sequence alignment. A putative ATP-binding site is shown in bold, compared to a consensus (Walker et al., 1982; Husain et al., 1986) in which $\mathrm{h}$ is a small hydrophobic residue and $\mathrm{X}$ is any residue. NBRF database accession numbers for BchI and Cs are S04401 (the former CrtA sequence, residues 242-591) and S08654, respectively.

characterization of the gene products derived from this region of the chromosome (Fig. 3).

Mutations in the $b c h D$ gene also disrupt the accumulation of Bchl or its immediate precursors (Zsebo \& Hearst, 1984; Giuliano et al., 1988). BchD is thought to be required either for insertion of magnesium or for the coupled methylation reaction required for the conversion of protoporphyrin IX into magnesium protoporphyrin IX monomethyl ester in Rhodobacter (Gorchein, 1973; Biel \& Marrs, 1983). The similarity of $\mathrm{BchI}^{-}$and BchD $^{-}$phenotypes (Zsebo \& Hearst, 1984; Giuliano et al., 1988) suggests that BchI may also be required for these coupled reactions.

Fig. 6 presents the alignment of the deduced amino acid sequences of BchI and the $A$. thaliana $\mathrm{Cs}$ proteins. The BchI protein displays $49 \%$ sequence identity with residues $70-424$ of $\mathrm{Cs}$, with similarity extending throughout the entire alignment. The predicted C-termini of the proteins align perfectly, while the $\mathrm{N}$-terminal extension of Cs, encoding the putative transit peptide, has no counterpart in BchI. Considering the enormous evolutionary distance between photosynthetic bacteria and higher plants, the striking degree of similarity predicted between BchI and Cs indicates that these proteins are homologues, and likely perform crucial functions.

Mutations in the nuclear-encoded $A$. thaliana $c s$ ( $c h$ 42) gene result in a pale pigmentation phenotype (Koncz et al., 1990), although no qualitative changes in pigment content were initally reported in a leaky T-DNA mutant (unpublished data cited in Koncz et al., 1990). The Cs protein appears to be involved in photosynthesis, based on the photosynthetically inviable phenotype of plants homozygous for the X-ray-induced $c h-42$ mutant allele of the $c s$ gene. A gene from the chloroplast genome of
Euglena gracilis, an alga, has also recently been found to encode a BchI/Cs-like product (Orsat et al., 1992). This observation indicates the export of the $c s(c h-42)$ gene from the chloroplast to the nucleus in the course of evolution from algae to higher plants.

The $R$. capsulatus, E. gracilis, and $A$. thaliana proteins all contain putative ATP-binding sites (Fig. 6). We note that chelation of magnesium into protoporphyrin IX is an ATP-requiring process in higher plants and that this reaction has been proposed to require at least two components, a soluble stromal protein and a membraneassociated protein (Castelfranco \& Beale, 1983; Walker \& Weinstein, 1991). It will be of interest to determine whether BchI/Cs-like gene products have been conserved in other photosynthetic organisms, and whether analogies exist between BchI and BchD from Rhodobacter and the two component system in higher plants.

\section{Conclusions}

The time courses of $R$. capsulatus crt and bch mRNA accumulation and promoter activities have been studied during a rigorous and well defined shift in growth conditions from aerobic respiration to anaerobic photosynthesis. The oxygen-regulated expression of many chromosomally clustered genes encoding pigment biosynthetic enzymes and pigment-binding polypeptides is temporally coordinated during the development of a photosynthetic membrane. A redetermination of the nucleotide sequence of $\operatorname{crtA}$ has revealed a frameshift, suggesting that the $3^{\prime}$ portion of this gene actually corresponds to $b c h I$. The predicted $b c h I$ gene product, required for Bchl synthesis or accumulation in Rhodobacter, is strongly conserved in more highly 
evolved photosynthetic organisms, including $E$. gracilis and $A$. thaliana.

We thank Drs G. Drews (Universität Freiburg, FRG) for supplying the plasmid $\mathrm{pRCl}$, Y.S. Zhu for invaluable advice on RNA isolation techniques, and E. Stutz (Université de Neuchatel, Switzerland) for communicating data prior to publication. We also thank $\mathrm{Dr} \mathrm{C}$. Maulbecker for critical reading of this manuscript. This material is based upon work supported by a National Science Foundation Graduate Fellowship to G.A., a National Institutes of Health training grant to D.C., and was also supported in part by National Institutes of Health grant GM 30786, and by the Office of Basic Energy Sciences, Biological Energy Division, Department of Energy under contract DEAC030-76SF00098.

\section{References}

Armstrong, G. A., Alberti, M., Leach, F. \& Hearst, J. E. (1989). Nucleotide sequence, organization, and nature of the protein products of the carotenoid biosynthesis gene cluster of Rhodobacter capsulatus. Molecular and General Genetics 216, 254-268.

Armstrong, G. A., Alberti, M. \& Hearst, J. E. (1990a). Conserved enzymes mediate the early reactions of carotenoid biosynthesis in nonphotosynthetic and photosynthetic prokaryotes. Proceedings of the National Academy of Sciences of the United States of America 87, 9975-9979.

Armstrong, G. A., Alberti, M., Leach, F. \& Hearst, J. E. (1990 $b$ ). Organization of the Rhodobacter capsulatus carotenoid biosynthesis gene cluster. In Molecular Biology of Membrane-Bound Complexes in Phototrophic Bacteria, pp. 39-46. Edited by G. Drews \& E. Dawes. New York: Plenum Press.

Armstrong, G. A., Schmidt, A., Sandmann, G. \& Hearst, J. E. $(1990 \mathrm{c})$. Genetic and biochemical characterization of carotenoid biosynthesis mutants of Rhodobacter capsulatus. Journal of Biological Chemistry 265, 8329-8338.

BAKER, M. E. \& FANESTIL, D. D. (1991). Mammalian peripheral-type benzodiazepine receptor is homologous to CrtK protein of Rhodobacter capsulatus, a photosynthetic bacterium. Cell 65, 721-722.

Bartley, G. E. \& ScolNiK, P. A. (1989). Carotenoid biosynthesis in photosynthetic bacteria. Journal of Biological Chemistry 264, 13109-13113.

Bartley, G. E., Schmidhauser, T. J., Yanofsky, C. \& Scolnik, P. A. (1990). Carotenoid desaturases from Rhodobacter capsulatus and Neurospora crassa are structurally and functionally conserved and contain domains homologous to flavoprotein disulfide oxidoreductases. Journal of Biological Chemistry 265, 16020-16024.

Belasco, J. G., Beatty, J. T., Adams, C. W., von Gabain, A. \& CoHEN, S. N. (1985). Differential expression of photosynthesis genes in $R$. capsulata results from segmental differences in stability within the polycistronic $r x c A$ transcript. Cell 40, 171-181.

BIEL, A. J. \& MARRS, B. L. (1983). Transcriptional regulation of several genes for bacteriochlorophyll biosynthesis in Rhodopseudomonas capsulata in response to oxygen. Journal of Bacteriology 156, 686-694.

BiEL, A. J. \& MARrs, B. L. (1985). Oxygen does not directly regulate carotenoid biosynthesis in Rhodopseudomonas capsulata. Journal of Bacteriology 162, 1320-1321.

Bramley, P. M. \& MACKENZIE, A. (1988). The regulation of carotenoid biosynthesis. In Current Topics in Cellular Regulation, vol. 29, pp. 291-343. Edited by B. L. Horecker \& E. R. Stadtman. London: Academic Press.

Burke, D. H., Alberti, M., Armstrong, G. A. \& Hearst, J. E. (1991 a). The complete nucleotide sequence of the $46 \mathrm{~kb}$ photosynthesis gene cluster of Rhodobacter capsulatus. EMBL Data Library, accession number Z11165.

Burke, D., Alberti, M., Stein, D. \& Hearst, J. E. (1991 b). Chlorophyll Fe proteins and other chlorophyll biosynthesis genes from Rhodobacter capsulatus to higher plants, abstract T/pm-B5. Abstract from the 19th Annual Meeting of the American Society for Photobiology. Photochemistry and Photobiology 53, 85S.
Castelfranco, P. A. \& Beale, S. I. (1983). Chlorophyll biosynthesis: recent advances and areas of current interest. Annual Review of Plant Physiology 34, 241-278.

Chamovitz, D., Misawa, N., Sandmann, G. \& HirschberG, J. (1992). Molecular cloning and expression in Escherichia coli of a cyanobacterial gene coding for phytoene synthase, a carotenoid biosynthesis enzyme. FEBS Letters 296, 305-310.

Chen, C.-Y. A., Beatty, J. T., Cohen, S. N. \& Belasco, J. G. (1988). An intercistronic stem-loop structure functions as an mRNA decay terminator necessary but insufficient for puf mRNA stability. Cell 52, 609-619.

Clark, W. G, Davidson, E. \& Marrs, B. L. (1984). Variations of mRNA levels coding for antenna and reaction center polypeptides in Rhodopseudomonas capsulata in response to changes in oxygen concentration. Journal of Bacteriology 157, 945-948.

Cogdell, R. J. \& Frank, H. A. (1987). How carotenoids function in photosynthetic bacteria. Biochimica et Biophysica Acta 895, 63-79.

Cohen-Bazire, G., Sistrom, W. R. \& Stanier, R. Y. (1957). Kinetic studies of pigment synthesis by non-sulfur purple bacteria. Journal of Cellular and Comparative Physiology 49, 25-68.

Cook, D. N., Armstrong, G. A. \& Hearst, J. E. (1989). Induction of anaerobic gene expression in Rhodobacter capsulatus is not accompanied by a local change in chromosomal supercoiling as measured by a novel assay. Journal of Bacteriology 171, 4836-4843.

Coomber, S. A., Chaudri, M., Connor, A., Britton, G. \& Hunter, C. N. (1990). Localized transposon Tn5 mutagenesis of the photosynthetic gene cluster of Rhodobacter sphaeroides. Molecular Microbiology 4, 977-989.

Demmig-Adams, B. (1990). Carotenoids and photoprotection in plants: a role for the xanthophyll zeaxanthin. Biochimica et Biophysica Acta $1020,1-24$.

DevereuX, J., Haeberli, P. \& Smithies, O. (1984). A comprehensive set of sequence analysis programs for the VAX. Nucleic Acids Research 12, 387-395.

Ditta, G., Stanfield, S., Corbin, D. \& Helinski, D. R. (1980). Broad host range DNA cloning system for Gram-negative bacteria: construction of a gene bank of Rhizobium meliloti. Proceedings of the National Academy of Sciences of the United States of America 77 , 7347-7351.

Giuliano, G., Pollock, D., Stapp, H. \& Scolnik, P. A. (1988). A genetic-physical map of the Rhodobacter capsulatus carotenoid biosynthesis gene cluster. Molecular and General Genetics 213, 78-83.

Golecki, J. R., Schumacher, A. \& Drews, G. (1980). The differentiation of the photosynthetic apparatus and the intracytoplasmic membrane in cells of Rhodopseudomonas capsulata upon variation of light intensity. European Journal of Cell Biology 23, 1-5.

GoodwIn, T. W. (1980). The Biochemistry of the Carotenoids, Vol. 1 : Plants, p. 63. New York: Chapman \& Hall.

GorCHEIN, A. (1973). Control of magnesium protoporphyrin chelatase activity in Rhodospseudomonas spheroides. Biochemical Journal 134, 833-845.

Hunter, C. N. \& CoOmber, S. A. (1988). Cloning and oxygenregulated expression of the bacteriochlorophyll biosynthesis genes bch $E, B, A$ and $C$ of Rhodobacter sphaeroides. Journal of General Microbiology 134, 1491-1497.

Husain, I., Van Houten, B., Thomas, D. C. \& Sancar, A. (1986). Sequences of Escherichia coli uvrA gene and protein reveal two potential ATP binding sites. Journal of Biological Chemistry 261, 4895-4901.

Kaufmann, N., Reidl, H. H., Golecki, J. R., Garcia, A. F. \& Drews, G. (1982). Differentiation of the membrane system in cells of Rhodopseudomonas capsulata after transition from chemotrophic to phototrophic growth conditions. Archives of Microbiology 131, 313-322.

KeISTER, D. L. (1978). Respiration versus photosynthesis. In The Photosynthetic Bacteria, pp. 849-856. Edited by R. K. Clayton \& W. R. Sistrom. New York: Plenum Press.

KILEY, P. J. \& KaPLAN, S. (1988). Molecular genetics of photosynthetic membrane biosynthesis in Rhodobacter sphaeroides. Microbiological Reviews 52, 50-69.

Klug, G., KaufmanN, N. \& Drews, G. (1985). Gene expression of 
pigment-binding proteins of the bacterial photosynthetic apparatus: transcription and assembly in the membrane of Rhodopseudomonas capsulata. Proceedings of the National Academy of Sciences of the United States of America 82, 6485-6489.

Koncz, C., Mayerhofer, R., KonCz-Kalman, Z., Nawrath, C., ReISS, B., REDEI, G. P. \& SCHELl, J. (1990). Isolation of a gene encoding a novel chloroplast protein by T-DNA tagging in Arabidopsis thaliana. EMBO Journal 9, 1337-1346.

Krinsky, N. I. (1971). Function. In Carotenoids, pp. 669-716. Edited by O. Isler, H. Gutmann \& U. Solms. Basel, Switzerland: Birkhäuser Verlag.

Leach, F., Armstrong, G. A. \& Hearst, J. E. (1991). Photosynthesis genes in Rhodobacter capsulatus can be regulated by oxygen during dark respiratory growth with DMSO. Journal of General Microbiology 137, 1551-1556.

MA, D., CoOK, D. N., O'Brien, D. A. \& Hearst, J. E. (1993). Analysis of the promoter and regulatory sequences of an oxygen-regulated $b c h$ gene in Rhodobacter capsulatus by site-directed mutagenesis. Journal of Bacteriology (in the press).

MARRS, B. (1981). Mobilization of the genes for photosynthesis from Rhodopseudomonas capsulata by a promiscuous plasmid. Journal of Bacteriology 146, 1003-1012.

Math, S. K., Hearst, J. E. \& Poulter, C. D. (1992). The crtE gene in Erwinia herbicola encodes geranylgeranyl diphosphate synthase. Proceedings of the National Academy of Sciences of the United States of America 89, 6761-6764.

Miller, J. H. (1972). Experiments in Molecular Genetics, pp. 352-355. Cold Spring Harbor, NY: Cold Spring Harbor Laboratory.

VAN Niel, C. B. (1944). The culture, general physiology, morphology and classification of the nonsulfur purple and brown bacteria. Bacteriological Reviews 8, 1-118.

OKa, A., SUgisaKi, H. \& M. TAKanami, M. (1981). Nucleotide sequence of the kanamycin resistance transposon Tn903. Journal of Molecular Biology 147, 217-226.

Orsat, B., Monfort, A., Chatellard, P. \& Stutz, E. (1992). Mapping and sequencing of an actively transcribed Euglena gracilis chloroplast gene (ccs A) homologous to the Arabidopsis thaliana nuclear gene $c s$ (ch-42). FEBS Letters 303, 181-184.

Sandmann, G. \& Misawa, N. (1992). New functional assignment of the carotenogenic genes $c r t B$ and $\operatorname{crt} E$ with constructs of these genes from Erwinia species. FEMS Microbiology Letters 90, 253-258.

SChUMACHER, A. \& Drews, G. (1978). The formation of bacteriochlorophyll-protein complexes of the photosynthetic apparatus of Rhodopseudomonas capsulata during the early stages of development. Biochimica et Biophysica Acta 501, 183-194.

Scolnik, P. A. \& MARRs, B. L. (1987). Genetic research with photosynthetic bacteria. Annual Review of Microbiology 41, $703-726$.

Straub, O. (1987). Key to Carotenoids, pp. 1-296. Edited by H. Pfander. Basel, Switzerland: Birkhäuser Verlag.
Taylor, D. P., Cohen, S. N., Clark, W. G. \& Marrs, B. L. (1983). Alignment of the genetic and restriction maps of the photosynthesis region of the Rhodopseudomonas capsulata chromosome by a conjugation-mediated marker rescue technique. Journal of Bacteriology 154, 580-590.

VIEIRA, J. \& Messing, J. (1982). The pUC plasmids, an M13mp7derived system for insertion mutagenesis and sequencing with universal primers. Gene 19, 259-268.

WALKER, C. J. \& WEINSTEIN, J. (1991). In vitro assay of the chlorophyll biosynthetic enzyme Mg-chelatase: Resolution of the activity into soluble and membrane-bound fractions. Proceedings of the National Academy of Sciences of the United States of America 88, 5789-5793.

Walker, J. E., Saraste, M., Runswick, M. J. \& Gray, N. J. (1982). Distantly related sequences in the $\alpha$ - and $\beta$-subunits of ATP synthase, myosin, kinases and other ATP-requiring enzymes and a common nucleotide binding fold. EMBO Journal 8, 945-951.

Wellington, C. L. \& BeatTy, J. T. (1989). Promoter mapping and nucleotide sequence of the $b c h C$ bacteriochlorophyll biosynthesis gene from Rhodobacter capsulatus. Gene 83, 251-261.

Wellington, C. L. \& BeATtY, J. T. (1991). Overlapping mRNA transcripts of photosynthesis gene operons in Rhodobacter capsulatus. Journal of Bacteriology 173, 1432-1443.

YANG, Z. \& BAUER, C. E. (1990). Rhodobacter capsulatus genes involved in early steps of the bacteriochlorophyll biosynthetic pathway. Journal of Bacteriology 172, 5001-5010.

Yen, H. C. \& Marrs, B. (1976). Map of genes for carotenoid and bacteriochlorophyll biosynthesis in Rhodopseudomonas capsulata. Journal of Bacteriology 126, 619-629.

Young, D. A., Bautr, C. E., Williams, J. C. \& Marrs, B. L. (1989). Genetic evidence for superoperonal organization of genes for photosynthetic pigments and pigment-binding proteins in Rhodobacter capsulatus. Molecular and General Genetics 218, 1-12.

YU, P. L., HoHN, B., FALK, H. \& DREWs, G. (1982). Molecular cloning of the ribosomal RNA genes of the photosynthetic bacterium Rhodopseudomonas capsulata. Molecular and General Genetics 188, 392-398.

ZHU, Y. S. \& HEARST, J. E. (1986). Regulation of the expression of the genes for light-harvesting antenna proteins LH-I and LH-II; reaction center polypeptides RC-L, RC-M, and RC-H; and enzymes of bacteriochlorophyll and carotenoid biosynthesis in Rhodobacter capsulatus by light and oxygen. Proceedings of the National Academy of Sciences of the United States of America 83, 7613-7617.

ZHU, Y. S. \& KAPLAN, S. (1985). Effects of light, oxygen, and substrates on steady-state levels of mRNA coding for ribulose-1,5-bisphosphate carboxylase and light-harvesting and reaction center polypeptides in Rhodopseudomonas sphaeroides. Journal of Bacteriology 162, 925-932.

ZSEBO, K. M. (1984). Genetic-physical mapping of a photosynthetic gene cluster in Rhodopseudomonas capsulata. $\mathrm{PhD}$ thesis, University of California, Berkeley, USA.

ZsEBO, K. M. \& HEARST, J. E. (1984). Genetic-physical mapping of a photosynthetic gene cluster from $R$. capsulata. Cell 37, 937-947. 